\title{
A space-time stochastic climatological approach to daily global solar radiation
}

\author{
Istvan Matyasovszky*, Istvan Bogardi \\ Civil Engineering Department, University of Nebraska - Lincoln, Lincoln, Nebraska 68588-0531, USA
}

\begin{abstract}
A stochastic climatological model is presented to describe the space-time behavior of daily global solar radiation measured by pyranometers. Due to the difficulty of describing daily global radiation by common probability distributions, a nonparametric technique, the Abramson kernel estimator, is used. The space-time climatological model of daily global radiation consists of a transformed multivariate autoregressive (AR) process conditioned on large-scale atmospheric circulation patterns. The transformation is necessary to establish a relationship between the probability distribution of global radiation and a normal variable used in the AR model. In order to check the stochastic model a simulation study was performed for 7 locations in Nebraska, USA. Although a split sampling approach could not be used due to the relatively small sample size the simulated probablity distributions reproduced the empirical distributions quite correctly. Also, the space-time dependency was maintained. The methodology may be a useful tool to estimate local/regional radiation under climate change.
\end{abstract}

KEY WORDS: Global solar radiation Probability distribution Kernel estimator - Space-time model . Circulation patterns . Downscaling

\section{INTRODUCTION}

The purpose of this paper is to develop a stochastic climatological model to describe the daily amount of global solar radiation (direct and diffuse incoming solar radiations). The model considers both the spatial and temporal dependency and is conditioned on a set of types of large-scale atmospheric circulation.

Basic meteorological elements, like temperature, precipitation, or wind speed, have been intensively examined concerning both their temporal and spatial behavior. However, no comprehensive statistical analysis has been performed for global radiation, although radiation is one of the most important meteorological factors. The spatial and temporal distribution of global radiation governs the main meteorological processes. For instance, temperature is highly dependent on incoming global radiation. A given portion

\footnotetext{
- On leave from Department of Meteorology, Eotvos Lorand University, Budapest, Hungary

E-mail: matya@ludens.elte.hu
}

of global radiation is utilized by evapotranspiration which has both meteorological and hydrological importance. This portion depends on surface albedo, water supply, wind speed, vegetation, and other parameters. Global radiation also has a key role in melting processes, an important factor of the hydrological cycle. Photosynthetically active radiation, the 0.4 to $0.7 \mu \mathrm{m}$ wavelength interval of direct radiation, is one of the principal factors governing the type and growth of vegetation.

The first step of a stochastic analysis is to model the underlying variable at a given location and time as a random variable. For instance, daily mean temperature may be represented as a normal variable, daily precipitation amount is described by a gamma distribution, or wind speed is modeled, among others, by a Weibull distribution (Essenwanger 1976). However, no similar model is available for daily global radiation. Therefore, the first goal of this paper is to estimate the probability distribution of daily global radiation. Daylight duration and cloudiness are the most important factors influencing global radiation. Daylight duration provides the 
characteristic annual cycle of global radiation at moderate and high latitudes, while cloudiness causes the high variability around its actual mean. Since also cloudiness has an annual cycle, the temporal variability of global radiation can be very complex. Mathematically speaking, the shape of the probability density function of global radiation can be very different for different periods. Therefore, the method for estimating the probability distribution of global radiation should be flexible. In this paper, a nonparametric approach, namely the so-called Abramson kernel estimator, is used to estimate the probability density.

Consecutive daily radiation values are statistically dependent, therefore, a time series model is necessary to reproduce this temporal dependency. For this purpose, first order autoregressive (AR) processes are used here. However, AR processes have been developed principally for Gaussian processes, but global radiation does not follow a Gaussian distribution. Therefore, a transformation establishing a relationship between the probability distributions of the global radiation and a normal random variable will be introduced. A natural way to describe the spatial variability of global radiation is to use multivariate AR processes when the components of the vector variable represent the locations.

Many studies have demonstrated that the stochastic behavior of meteorological elements can be considered as a result of large-scale forcing and local effects (Lamb 1977, Sowden \& Parker 1981, Bardossy \& Caspary 1990, Leathers 1991, Leathers et al. 1991). Forcing can be characterized by typical large-scale circulation patterns (CPs) of the atmosphere and the local influence can be described by linking the meteorological variables to $\mathrm{CP}$ types. Specifically, the parameters of the multivariate AR process are CP type dependent.

In recent years considerable effort has focused on both the methodology and application of downscaling low spatial resolution output of General Circulation Models (GCMs). Attention is directed principally to temperature and precipitation; the space-time model developed in this paper can be used for downscaling daily global radiation by substituting the observed $\mathrm{CP}$ data with GCM-generated CP data.

The paper is organized as follows. First, the radiation and CP data sets are defined. Then the probability distribution of daily global radiation is estimated after a brief theoretical overview of kernel estimators. The conditional multivariate AR model is introduced and discussed in the next section. The stochastic model is then applied with 7 stations by a simulation study comparing the observed and simulated statistics. The possibility of using the model for downscaling purposes is outlined. Finally, a section for discussion and conclusions is provided.

\section{DATA SETS}

Seven automated weather stations having the longest records were selected in Nebraska, USA (Fig. 1). The data sets available from the Nebraska State Climate Office, High Plains Climate Center cover a relatively short common period from July 1, 1982 to December 31, 1993.

Geopotential data are represented by the National Meteorological Center (NMC) grid point analyses of the $700 \mathrm{hPa}$ pressure surfaces available from the National Center for Atmospheric Research (NCAR). The analysis is based on daily values (12:00 h UTC) at 40 points on a diamond grid covering the sector $25^{\circ}$ to $60^{\circ} \mathrm{N}, 80^{\circ}$ to $125^{\circ} \mathrm{W}$ for the period January 1962 through June 1989. This data set is used to classify daily CPs in order to develop a set of daily types. Note that the conditional space-time stochastic model is developed using data sets from July 1, 1982 to June 30 , 1989 , which is a relatively short period for a stochastic analysis including split sampling.

Classification schemes of spatial meteorological variables are often based on macrocirculation patterns characterized by the spatial distribution of either sea level air pressure, or low or middle level tropospheric pressure heights. There are several possibilities combined with considerable experience for classifying daily CPs. One of the main approaches includes subjective classifications when meteorological experience is applied. These classifications are subjective because they reflect the subjectivity of meteorologists, but are objective in the sense that they are based on the physical be-

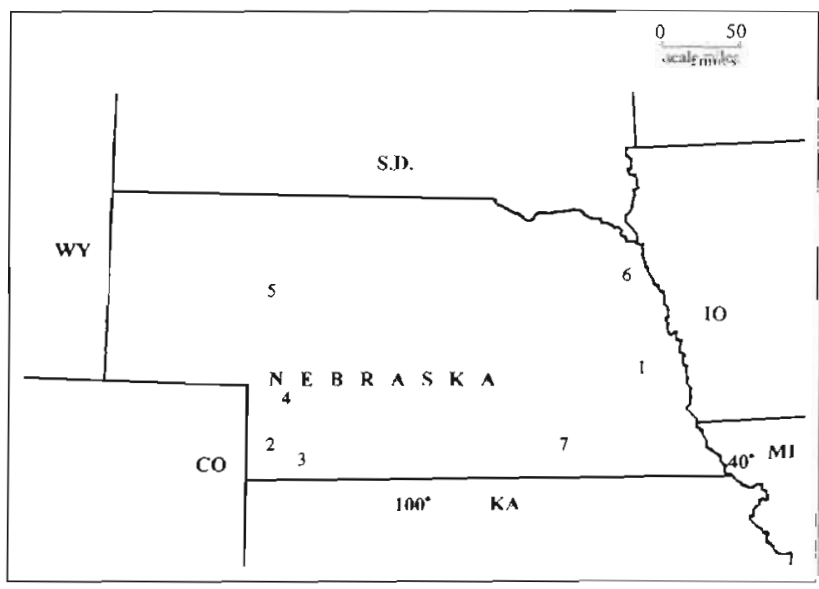

Fig. 1. Radiation measurement stations: coordinates of locations used in the analysis: 1 , Mead (latitude $41.08^{\circ} \mathrm{N}$, longrtude $96.30^{\circ} \mathrm{W}$, elevation $\left.366 \mathrm{~m}\right) ; 2$, Champion $\left(40.23^{\circ} \mathrm{N}\right.$, $\left.101.43^{\circ} \mathrm{W}, 1029 \mathrm{~m}\right) ; 3, \operatorname{McCook}\left(40.14^{\circ} \mathrm{N}, 100.35^{\circ} \mathrm{W}, 729 \mathrm{~m}\right)$; 4 , Dickens $\left(41.00^{\circ} \mathrm{N}, 100.56^{\circ} \mathrm{W}, 945 \mathrm{~m}\right) ; 5$, Arthur $\left(41.39^{\circ} \mathrm{N}\right.$, $\left.100.31^{\circ} \mathrm{W}, 1097 \mathrm{~m}\right) ; 6$, West Point $\left(41.49^{\circ} \mathrm{N}, 96.49^{\circ} \mathrm{W}, 442 \mathrm{~m}\right)$; 7. Clay Center $\left(40.32^{\circ} \mathrm{N}, 98.09^{\circ} \mathrm{W}, 552 \mathrm{~m}\right)$ 
havior of the atmosphere. Baur et al. (1944) defined 29 types for Europe: a list of these circulation patterns and the corresponding meteorological descriptions can be found in Hess \& Brezowsky (1969). Another classification scheme for the extratropical latitudes of the Northern Hemisphere was developed in Dzerdzeevskii (1968). In contrast, Lamb (1972) introduced circulation patterns for the British Isles, a relatively small area.

Recently, high-speed computers and the appearance of modern mathematical clustering algorithms have made it possible to use so-called objective classification methods (Hartigan 1975). Objectivity means here that a given algorithm makes the data processing automatic, although the choice of algorithm includes subjectivity. The so-called hierarchical algorithms are relatively simple, but need large computer storage even for moderate sample sizes since each sample element is handied as a possible cluster. The most common techniques are based on correlation coefficients or sum of squared differences of maps (Lund 1963, Kirchofer 1973). A mathematically well-developed and computerized nonhierarchical technique is the $k$-means method (MacQueen 1967), but in recent years, principal component analysis (PCA) (Craddock \& Flood 1969, Kutzbach 1970) and PCA coupled with clustering (Key \& Crane 1986) have been preferred.

In the present paper a $k$-means algorithm is used on the basis of an Euclidian distance of daily CPs. Before using the $k$-means method a PCA is performed because a conjunctive use of these techniques usually provides the most separable system of clusters with the most concentrated clusters (Gong \& Richman 1992). The periods from April to September (summer) and from October to March (winter) were examined separately, and pressure height values at each grid point are standardized to exclude the annual cycle. The number of $\mathrm{CP}$ types was chosen as a compromise between the increasing number of clusters and the decreasing sum of inner distances within each cluster. The inner distance of a given cluster is defined as the mean of squared differences between the cluster center and cluster elements. Using this concept, 9 types for both winter and summer seems to be a good compromise in the present case. The relative frequencies of $\mathrm{CP}$ types are shown in Table 1; a more detailed description of $\mathrm{CP}$ types can be found in Matyasovszky $\&$ Bogardi (1996).

Table 1. Relative frequency of CP types

\begin{tabular}{|llllllllll|}
\hline & $\mathrm{CP1}$ & $\mathrm{CP} 2$ & $\mathrm{CP} 3$ & $\mathrm{CP} 4$ & $\mathrm{CP} 5$ & $\mathrm{CP} 6$ & $\mathrm{CP} 7$ & $\mathrm{CP} 8$ & $\mathrm{CP9}$ \\
\hline Summer & .135 & .096 & .090 & .082 & 116 & .105 & 184 & .108 & .084 \\
Winter & .099 & .185 & .068 & .093 & .120 & .094 & .097 & .121 & .123 \\
\hline
\end{tabular}

\section{ESTIMATION OF THE PROBABILITY DISTRIBUTION}

The Parzen-Rosenblatt kernel density estimate, $\hat{f}(x)$, from a sample $\left\{x_{1}, x_{2}, \ldots, x_{n}\right\}$ of size $n$ is given by

$$
\hat{f}(x)=\frac{1}{n} \sum_{i=1}^{n} \frac{1}{b} K\left(\frac{x-x_{i}}{b}\right)
$$

where $K(z)$ is a so-called kernel function satisfying certain properties to provide an appropriate estimate of $f(x)$ (Rosenblatt 1956, Parzen 1962). The bandwidth $b$ tends to zero as $n$ tends to infinity.

A possibility for choosing the kernel is that $K(z)$ itself is a density function because $K(z)$ is required to integrate to unity. $K(z)$ with support $[-1,1]$ is called a kernel of order $k$ if the condition

$$
\int_{-1}^{1} K(z) z^{j} \mathrm{~d} z= \begin{cases}1, & j=0 \\ 0, & 0<j<k\end{cases}
$$

is satisfied

The choice of kernels is based on the asymptotic properties of the estimator (Eq. 1). Minimum variance kernels minimizing the asymptotic variance of Eq. (1) can be found in Gasser et al. (1985). Muller (1984) discussed a more general class of kernel functions which minimizes the variance of the $\lambda$ th $(\lambda \geq 0)$ derivative of the kernel estimate. All the above kernels, however, may be used only in the case when $f(x)$ is defined over the interval $-\infty$ to $\infty$, unless the kernels are modified near the endpoints. Muller (1991) has developed a very general formulation to have kernels applicable for any density at any $x$ and for any $k$.

In parametric density estimation, the parameters are estimated by maximum likelihood (ML), least squares (LS), or other methods. A natural way to estimate the bandwidth is, therefore, to use these concepts for kernel estimators. The LS for estimating $b$ based on a minimization of the cross-validated integrated mean square error (IMSE) of Eq. (1) was suggested by Rudemo (1982). Hall \& Marron (1987) demonstrated the optimality of LS for density estimation in terms of IMSE. They showed that no other bandwidth selection procedure can deliver smaller IMSE than the crossvalidated LS.

Mean square error of $\hat{f}(x)$ is governed by $f(x)$ and $f^{(k)}(x)$ [the $k$ th derivative of $f(x)$ ]. This fact motivates the choice of locally varying bandwidths. A smaller bandwidth near the peaks of $f(x)$ reduces bias and a larger bandwidth in the flat regions of $f(x)$ reduces variance. Therefore, it may be expected that a proper strategy for choosing local bandwidth, $b(x)$, yields smaller IMSE than the IMSE stemming from ordinary bandwidth selection for global bandwidth, $b$. In general, the construction of a local bandwidth estimator entails a 2step procedure. The first step produces a pilot estima- 
tor, $\hat{f}$, using a fixed bandwidth and the second stage yields the local bandwidth estimator. This second step requires a reformulation of $\mathrm{Eq}$. (1). A frequently used type of varying bandwidth is the so-called Abramson estimator defined by

$$
\check{f}(x)=\frac{1}{n} \sum_{i=1}^{n} \frac{\hat{f}^{1 / 2}\left(x_{i}\right)}{h} K\left(\frac{\left(x-x_{i}\right) \hat{f}^{1 / 2}\left(x_{j}\right)}{h}\right)
$$

because the choice $b\left(x_{i}\right)=h f^{-1 / 2}\left(x_{i}\right)$ eliminates the asymptotic bias of $\check{f}$. The parameter $h$ in Eq. (2) can be estimated by cross-validation as described by Hall (1992)

The Abramson technique with Muller's (1991) kernel under $k=2$ and $\lambda=1$ was used to estimate the probability density function of daily global radiation. Fig. 2 shows, as an example, the histogram and the estimated density at Mead in July and January. Note that the densities are smooth enough to satisfy a subjective smoothness property required for any estimate of probability distributions, while they fit the histogram accurately. The sharp peak of the densities at high radiation values corresponds to clear or slightly cloudy sky, while a second smaller mode at moderate and low radiation values is due to overcast sky.

\section{CONDITIONAL MODELING ON CP TYPES}

\subsection{Conditional probability distribution}

The probability distribution of daily global radiation will be formed as a superposition of probability distributions conditioned on CP types. Because radiation has a strong annual cycle (Fig. 3) relatively short periods should be analyzed separately within the year. However, if the density function is estimated, for instance, on a monthly basis the number of observations available for different $\mathrm{CP}$ types is very small and the estimation of the density can be highly inaccurate. Therefore, we assume that the shape of the density is 'similar' under longer periods (say seasons) and a 'universal' density can be defined for each season. Taking a simple example, the density function $f(x)$ of a normal variable having expected value $m$ and standard deviation $d$ can be obtained as $f(x)=1 / d \cdot \varphi((x-m) / d)$ where $\varphi$ is the density function of the standard normal variable (zero expected value, unit standard deviation). In our terminology, therefore, the density $\varphi$ is universal for normal distributions. In the present case, the universal density is estimated by the Abramson technique after an appropriate transformation of original data. The inverse of this transformation, then, results in the final estimate for each day.

Fitting sine and cosine waves of 1 yr and $1 / 2$ yr periods to radiation data the mean, $\mu(t)$, and standard
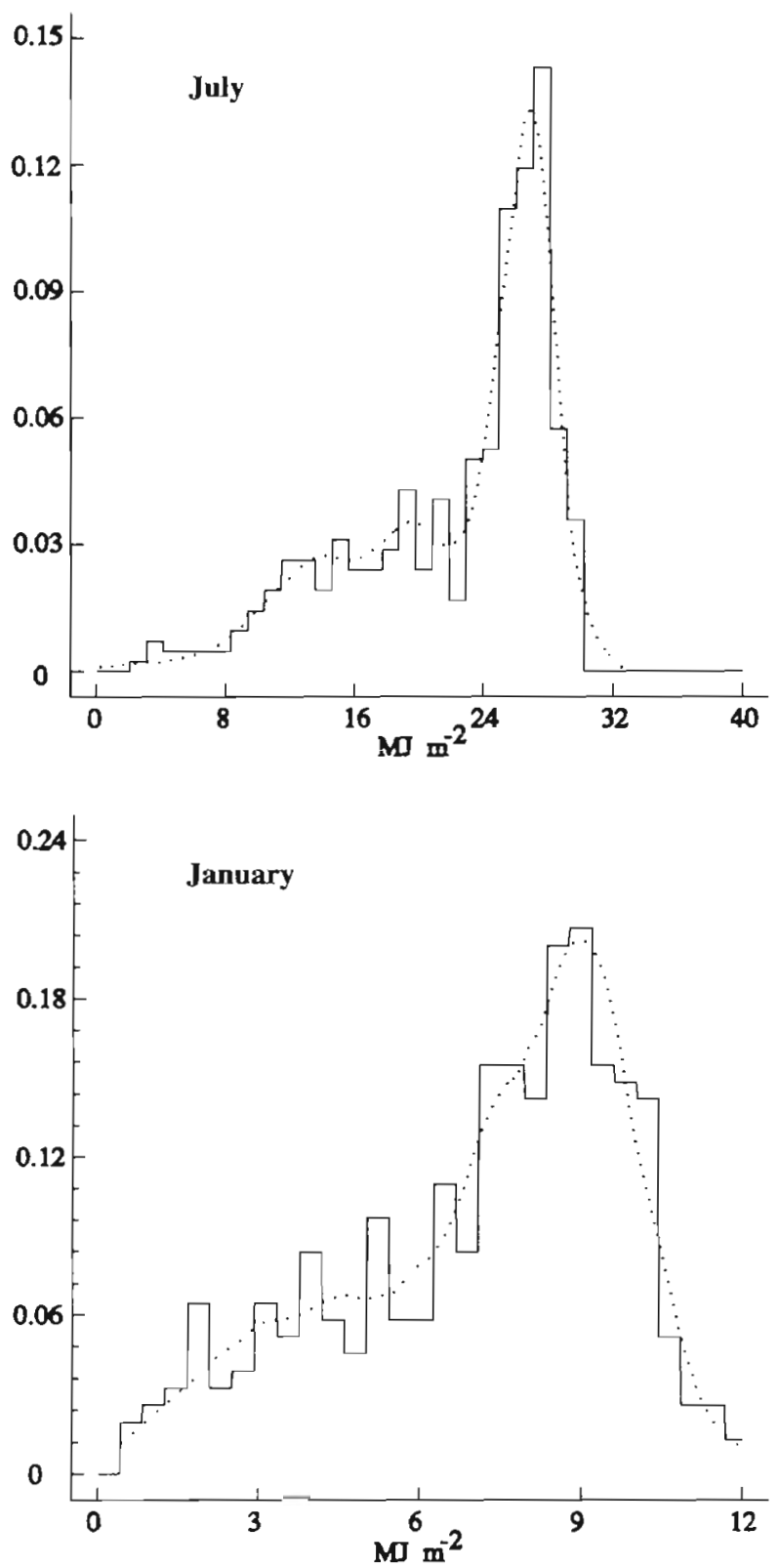

Fig. 2. Histogram (solid line) and estimated probability density function (dotted line) of daily global radiation at Mead in July (top) and January (bottom)

deviation, $\sigma(t)$, can be estimated. Fig. 3 shows the annual cycle of the mean and standard deviation. These curves are used to standardize the radiation data set $x_{1}, x_{2}, \ldots, x_{r}$ by

$$
y_{t}=\left(x_{t}-\hat{\mu}_{t}\right) / \hat{\sigma}(t), \quad t=1,2, \ldots, T
$$

Taking those standardized data which are accompanied with the same CP type in a given season a subset of the original data is obtained for each CP type in each season. The density function $g_{j}(y)$ for CP type $j$ in a season is taken universal and is estimated by using the 


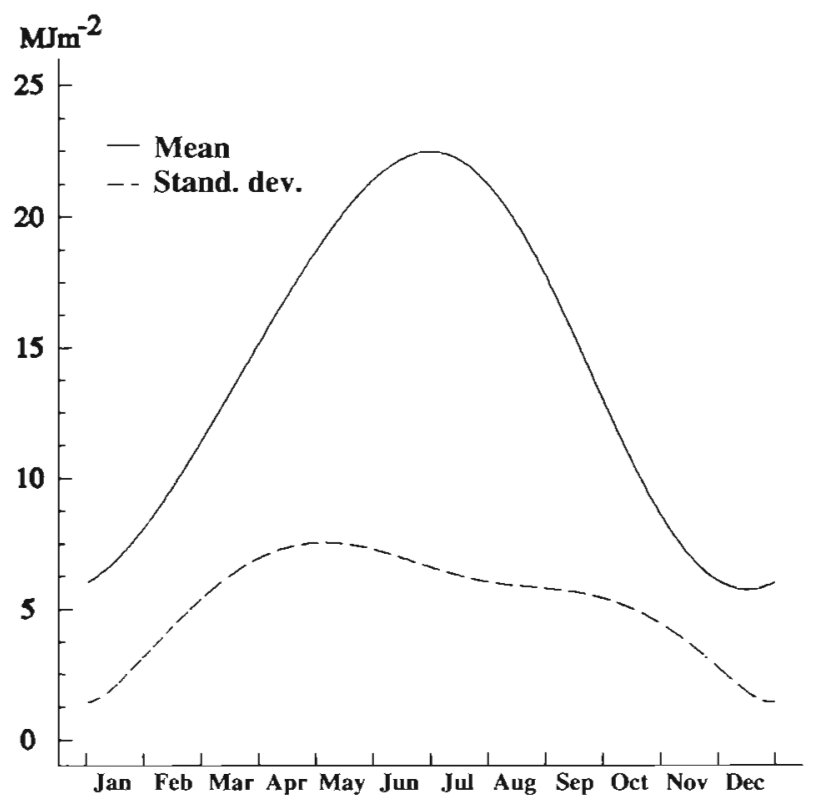

Fig. 3. Annual cycle of daily global radiation at Mead

Abramson technique for each data subset. Then the corresponding probability density function $f_{j}(x)$ of daily global radiation for day $t$ is estimated as

$$
\hat{f}_{j}(x)=1 / \hat{\sigma}(t) \cdot \check{g}_{j}(x-\hat{\mu}(t)) / \hat{\sigma}(t)
$$

Fig. 4 shows the probability density function of daily global radiation for June 15 and January 15 under 3 different CP types. These dates, typical days of summer and winter seasons, represent the almost largest and smallest average radiations in the year. In summer, the densities are similar in the sense that the most probable values of radiation appear almost the same, but the magnitude of the peaks and thus the width of the densities are highly different. In the other words, the most probable radiation values are independent from the type of large-scale circulation, but the variance of radiation are depend on circulation. This latter phenomenon is obviously a consequence of the cloudiness variability corresponding to different $C P$ types. In winter, the densities differ substantially, one of the CP types exhibits strong bimodality. Comparing the 2 seasons, CP types in summer can separate the behavior of radiation in a smaller degree since the cloudiness arising from convective effects decreases the role of different types of large-scale circulation.

\subsection{Space-time stochastic model}

To reproduce the space-time statistical structure of local climatic factors, a suitable model should be chosen. Autoregressive processes represent a welldeveloped and commonly used tool to model time series. They have been developed principally for Gaussian processes, but global radiation does not follow a Gaussian distribution. Therefore, it is desirable to construct a transformation establishing a relationship between the distribution of the global radiation and a normal distribution.

For mathematical formulation some notations are introduced as follows: $\alpha_{\ell}$ having possible values $j=$ $1,2, \ldots, J$ represents the CP type as a random variable at time $t$ ( $J$ is the number of types). Furthermore, let the vector $X(t)=\left(X\left(t, u_{1}\right), X\left(t, u_{2}\right), \ldots, X\left(t, u_{K}\right)\right)$ represent the
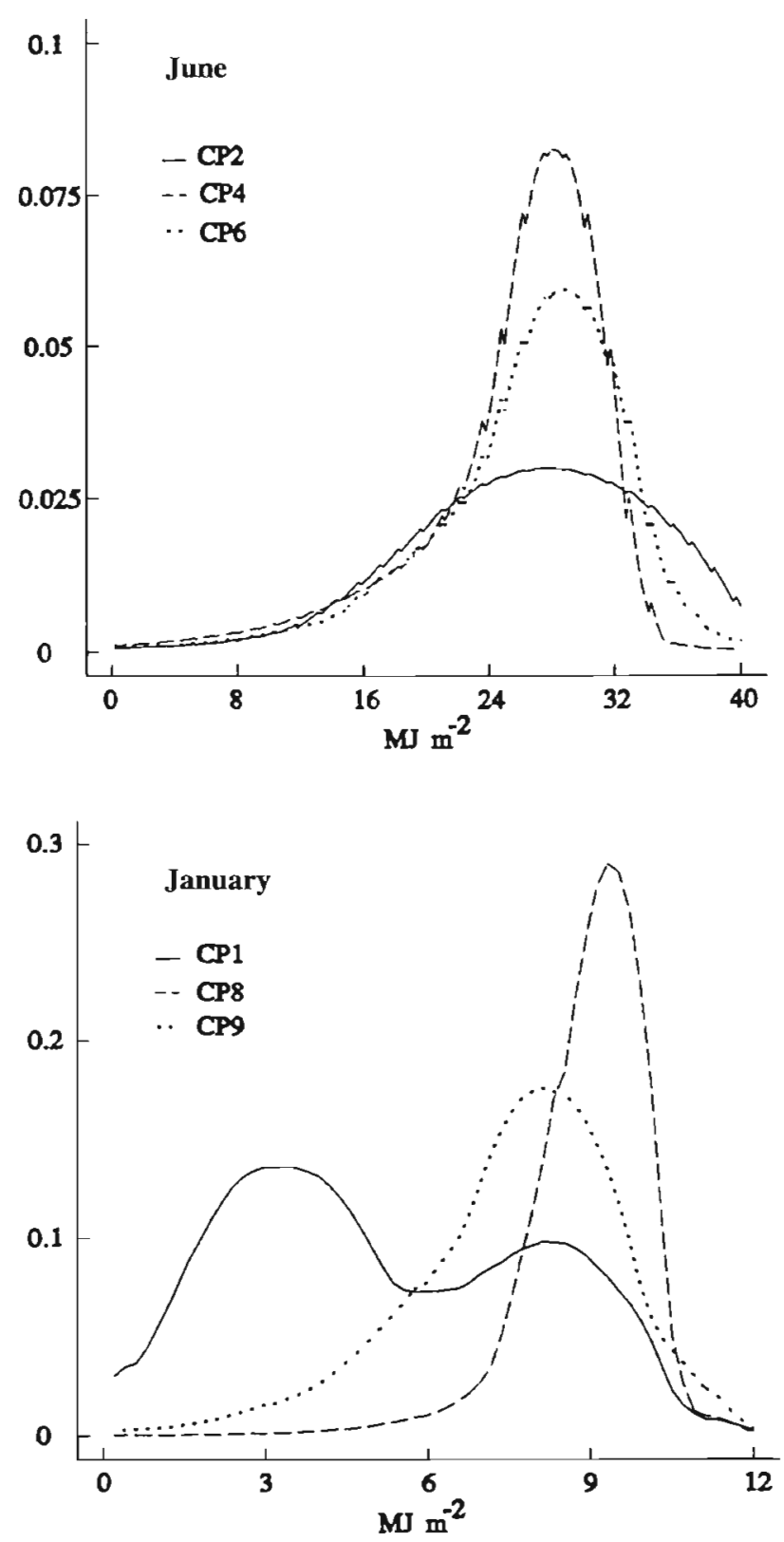

Fig. 4. Estimated probability density function of daily global radiation conditioned on different $C P$ types at Mead on June 15 (top) and January 15 (bottom) 
daily global radiation at locations $u_{\imath}, u_{2}, \ldots, u_{K}$ and time $t$ and let $\boldsymbol{W}(t)$ be a $K$-dimensional normal random vector at time $t$. We suppose for simplicity that each component of the vector $\boldsymbol{W}(t)$ has unit variance. The temporal evolution of $\boldsymbol{W}(t)$ is modeled by first-order autoregressive $[\mathrm{AR}(1)]$ processes.

Any component of daily global radiation $X$ of $X(t)$ for CP type $j$ can be calculated from the corresponding component $w$ of $\boldsymbol{W}(t)$ as

$$
X=F_{j}^{-1}(\Phi(w))
$$

where $F_{j}(x)$ is the distribution function of global radiation for CP type $j$ corresponding to the density (Eq. 3), and $\Phi$ is the standard normal distribution function. When CP type $j$ occurs at time $t$, $\boldsymbol{W}(t)$ is described by an AR(1) process defined as:

$$
\boldsymbol{W}(t)=\boldsymbol{B}_{j} \boldsymbol{W}(t-1)+\boldsymbol{C}_{j} \boldsymbol{U}(t)
$$

Using the multivariate version of the socalled Yule-Walker equations (Priestley 1981), the matrices $\boldsymbol{B}_{j}$ and $\boldsymbol{C}_{j}$ can be calculated from

$$
\begin{gathered}
\boldsymbol{B}_{j}=\boldsymbol{G}_{1 j} G_{0 j}^{-1} \\
C_{j} C_{j}^{T}=G_{0 j}-G_{1 j} G_{0 j}^{-1} G_{1 j}^{T}
\end{gathered}
$$

where the superscript $T$ denotes transpose and the conditional expectations $E\left[\boldsymbol{W}(t) \boldsymbol{W}(t-i) \mid \alpha_{t}=j\right], \quad i=0,1$ are the covariance matrices $G_{j j}$ of $\boldsymbol{W}(t)$ for lags $i=0,1$ and CP type $j . \quad U(t)$ represents a $K$-dimensional standard normal variable which consists of $K$ standard normal uncorrelated random variables. The matrices $G_{0,}, G_{1}$ can be estimated from radiation data using correlations of radiation indicator series as proposed for daily precipitation in Bardossy \& Plate (1992).

\section{APPLICATION}

The space-time stochastic model of daily radiation is applied with simulation techniques to the 7 stations in the following way. First, a set of circulation patterns has to be selected. This may be an observed data set, but circulation patterns may also be generated by simulation using a Markov chain model (Bardossy \& Plate 1990, Bogardi et al. 1992). Generation of a time series of process $W(t)$ and thus $\boldsymbol{X}(t)$ starts with the simulation of the white noise process $U(t)$. Eq. (5) is then used to calculate $\boldsymbol{W}(t)$. Finally, the transformation Eq. (4) is used for each component of $\boldsymbol{W}$ to obtain a daily global radiation vector $\boldsymbol{X}$. These steps are repeated until a time series length applicable for statistical analysis is achieved. This length is chosen to be equal to the observed data set length.

Radiation is variable both in space and time. The 7 stations represent 2 regions, namely Stns 1,6 and 7 are located in eastern Nebraska and Stns 2, 3, 4 and 5 in western Nebraska (Fig. 1). Table 2 shows correlations of global radiation among stations. As is

\begin{tabular}{|c|c|c|c|c|c|c|c|}
\hline \multicolumn{8}{|c|}{ July } \\
\hline \multicolumn{8}{|c|}{ Observed } \\
\hline 1 & 1.000 & 0.490 & 0.573 & 0.601 & 0.408 & 0.777 & 0.689 \\
\hline 2 & & 1.000 & 0.830 & 0.867 & 0.688 & 0.370 & 0.524 \\
\hline 3 & & & 1.000 & 0.862 & 0.562 & 0.383 & 0.577 \\
\hline 4 & & & & 1.000 & 0.773 & 0.482 & 0.611 \\
\hline 5 & & & & & 1.000 & 0.536 & 0.554 \\
\hline 6 & & & & & & 1.000 & 0.728 \\
\hline 7 & & & & & & & 1.000 \\
\hline \multicolumn{8}{|c|}{ Simulated } \\
\hline & 1 & 2 & 3 & 4 & 5 & 6 & 7 \\
\hline 1 & 1.000 & 0.423 & 0.477 & 0.432 & 0.490 & 0.888 & 0.695 \\
\hline 2 & & 1.000 & 0.768 & 0.763 & 0.701 & 0.404 & 0.555 \\
\hline 3 & & & 1.000 & 0.748 & 0.632 & 0.450 & 0.600 \\
\hline 4 & & & & 1.000 & 0.875 & 0.383 & 0.585 \\
\hline 5 & & & & & 1.000 & 0.469 & 0.629 \\
\hline 6 & & & & & & 1.000 & 0.670 \\
\hline 7 & & & & & & & 1.000 \\
\hline \multicolumn{8}{|c|}{ January } \\
\hline \multicolumn{8}{|c|}{ Observed } \\
\hline & 1 & 2 & 3 & 4 & 5 & 6 & 7 \\
\hline 1 & 1.000 & 0.476 & 0.527 & 0.490 & 0.456 & 0.938 & 0.824 \\
\hline 2 & & 1.000 & 0.875 & 0.865 & 0.852 & 0.489 & 0.666 \\
\hline 3 & & & 1.000 & 0.902 & 0.831 & 0.508 & 0.723 \\
\hline 4 & & & & 1.000 & 0.912 & 0.473 & 0.677 \\
\hline 5 & & & & & 1.000 & 0.461 & 0.621 \\
\hline 6 & & & & & & 1.000 & 0.806 \\
\hline 7 & & & & & & & 1.000 \\
\hline \multicolumn{8}{|c|}{ Simulated } \\
\hline & 1 & 2 & 3 & 4 & 5 & 6 & 7 \\
\hline 1 & 1.000 & 0.556 & 0.660 & 0.585 & 0.529 & 0.927 & 0.783 \\
\hline 2 & & 1.000 & 0.853 & 0.882 & 0.829 & 0.615 & 0.697 \\
\hline 3 & & & 1.000 & 0.897 & 0.775 & 0.715 & 0.789 \\
\hline 4 & & & & 1.000 & 0.906 & 0.646 & 0.755 \\
\hline 5 & & & & & 1.000 & 0.580 & 0.711 \\
\hline 6 & & & & & & 1.000 & 0.833 \\
\hline 7 & & & & & & & 1.000 \\
\hline
\end{tabular}
expected, stations within regions have high correlations while low correlations are observed between

Table 2 . Correlations of daily global radiation among stations 
regions. Both the correlation values and the difference between high and low correlations are somewhat smaller in July than January due to the stronger local effects (convective activity). The simulated correlation matrices reproduce these characteristics.

Autocorrelations with a confidence interval for the $95 \%$ probability level are shown in Fig. 5. In July, the autocorrelations indicate a weak but long 'memory' of daily global radiation. The simulation underestimates the autocorrelations for Days 1 to 5 , but overestimates them after the fifth day. January exhibits smaller autocorrelations; only a small correlation for 1 day lag should be considered signifi-
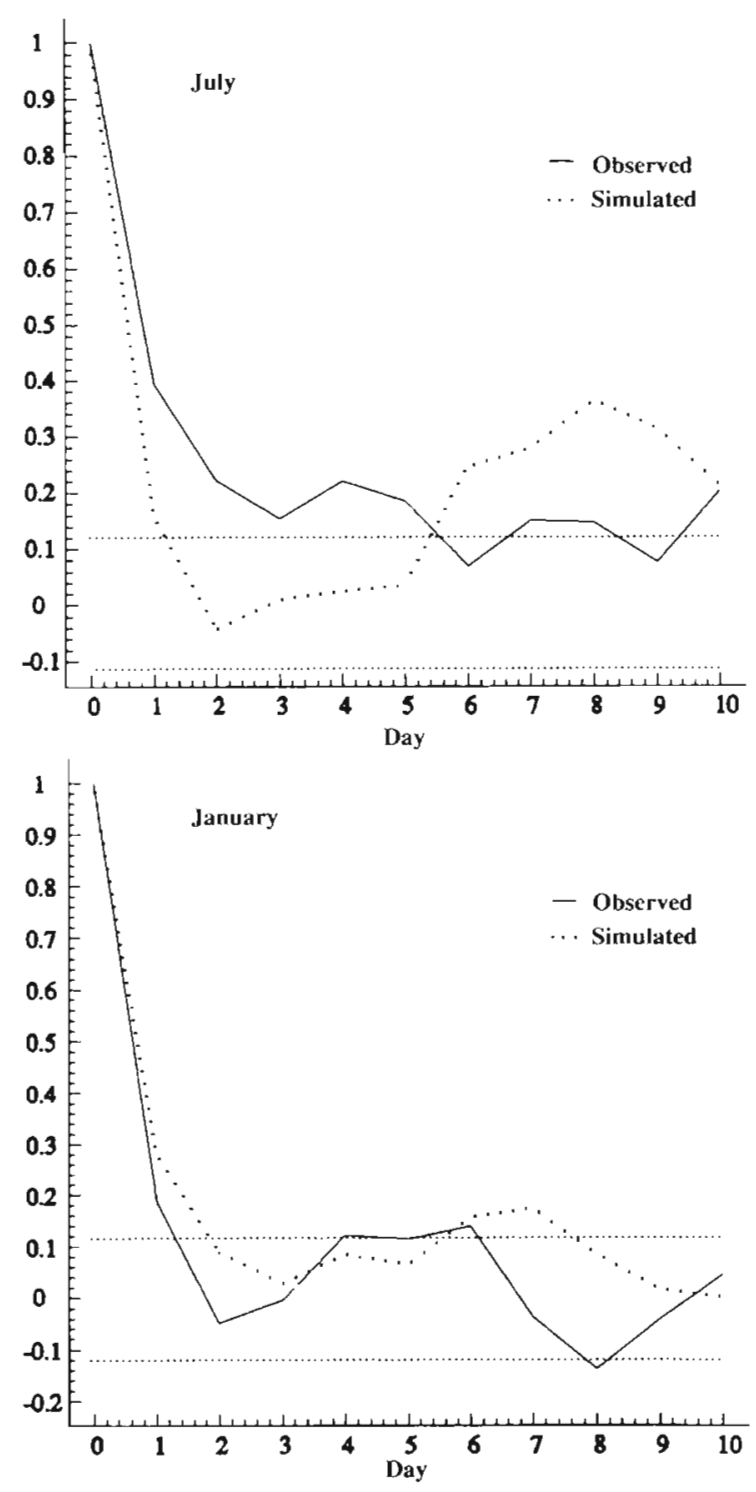

Fig. 5. Observed and simulated autocorrelations of daily global radiation at Mead in July (top) and January (bottom)
Table 3. Comparison of observed and simulated statistics of daily global radiation

\begin{tabular}{|lcccc|}
\hline & \multicolumn{2}{c}{ July } & \multicolumn{2}{c}{ January } \\
& Obs. & Simul. & Obs. & Simul. \\
\hline Mean $\left(\mathbf{M J ~ m}^{-2}\right)$ & & & & \\
Stn 1 & 23.5 & 24.1 & 7.2 & 6.8 \\
Stn 2 & 25.6 & 26.2 & 8.4 & 7.9 \\
Stn 3 & 25.3 & 26.5 & 8.9 & 8.5 \\
Stn 4 & 25.1 & 25.7 & 8.4 & 8.0 \\
Stn 5 & 25.0 & 25.4 & 8.1 & 7.8 \\
Stn 6 & 24.2 & 25.1 & 7.4 & 6.9 \\
Stn 7 & 24.2 & 24.9 & 7.9 & 7.3 \\
& & & & \\
Standard deviation (MJ $\left.\mathbf{m}^{-2}\right)$ & & & \\
Stn 1 & 5.8 & 5.8 & 2.4 & 3.2 \\
Stn 2 & 4.8 & 4.5 & 2.4 & 3.4 \\
Stn 3 & 5.4 & 4.6 & 2.6 & 3.4 \\
Stn 4 & 5.4 & 5.0 & 2.4 & 3.5 \\
Stn 5 & 5.2 & 5.1 & 2.3 & 3.8 \\
Stn 6 & 5.5 & 5.0 & 2.5 & 3.1 \\
Stn 7 & 4.6 & 4.3 & 2.6 & 3.6 \\
\hline
\end{tabular}

cantly different from zero. This almost negligible memory is due to the cyclonic activity which is much stronger in the winter season than in summer. Simulated autocorrelations fit the observed values satisfactorily. Probably, using a higher order AR process the autocorrelations could be reproduced also in summer, but then the number of parameters to be estimated would be too large when using the current short data set. The choice of AR(1) process is a compromise between model accuracy and number of model parameters.

Table 3 summarizes a comparison of observed and simulated means and standard deviations of daily global radiation. Although the mean is somewhat overestimated in July and underestimated in January the bias does not look considerable. Simulated means fit the observed means especially well in July. The standard deviation is also reproduced properly in July, but is overestimated in January. The observed and simulated probability distribution functions (Fig. 6) strengthen the above facts.

The origin of inaccurate reproduction of observed standard deviation in January is related to the procedure of radiation data standardization, density estimation and transformation of these densities. The standardization provides homogeneity in the mean and standard deviation of the standardized data, but does not yield identical probability distributions of those data. Probably, even the season is too long a period to obtain truly universal densities. The actual length of radiation data available in the present case, however, does not make it possible to use considerably shorter periods than seasons. 

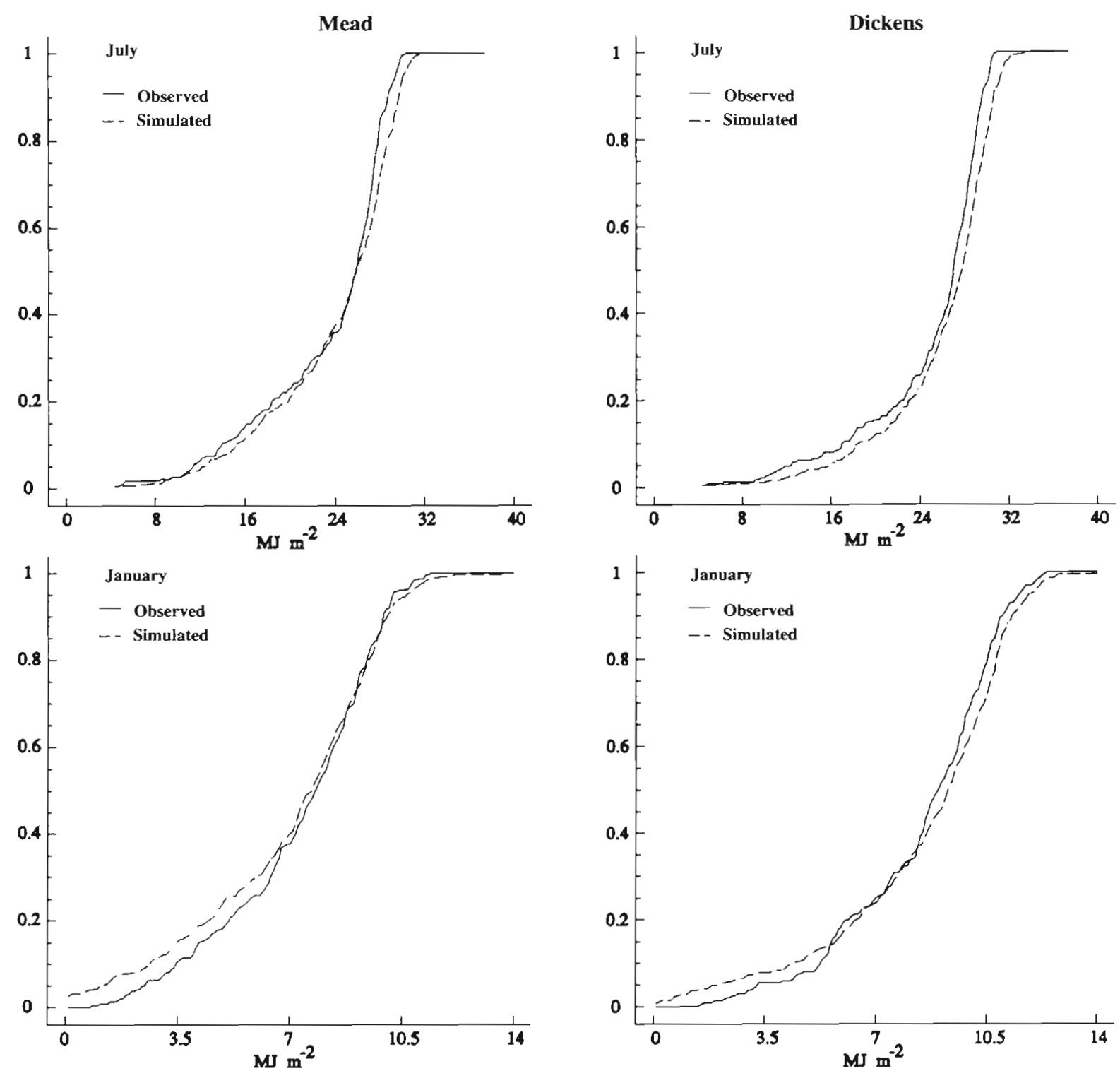

Fig. 6. Observed and simulated probability distribution functions of daily global radiation at Mead (left) and Dickens (right) in July (top) and January (bottom)

\section{POSSIBILITIES FOR DOWNSCALING}

A possible application of the presented methodology is to downscale the low spatial resolution output of General Circulation Models (GCMs). Various downscaling techniques have been developed to overcome the scale differences between GCM outputs and local climatic variables (Giorgi \& Mearns 1991). One of these techniques includes the so-called CP type approach used mostly with precipitation (Bardossy \& Plate 1992, Wilson et al. 1992, Matyasovszky et al. 1993a) and temperature (Matyasovszky et al. 1994, Easterling 1995). However, daily solar radiation could be also downscaled using the stochastic model presented. Since the most reliable output of GCMs found to be the large-scale daily atmospheric circulation patterns (e.g. Simmons \& Bengtsson 1988) the idea is to use only this GCM information as follows. Every GCM-generated daily CP is assigned to one of the CP types defined on observed CPS. The similarity of daily CPs is based on an Euclidian distance as in the case of observed data. Thus, a set of GCM-generated CP types is obtained. This set can be used directly or a new set of types can be simulated after fitting a Markov chain to the original set of CP types. Then, the space-time model is applied with the simulation procedure using daily $\mathrm{CP}$ types corresponding to the GCM output. 
The most important concerns regarding this type of downscaling include: (1) sensitivity of results on classification schemes and the number of CP types, (2) the dilemma of whether CP types obtained from observed data may be used for GCM output, i.e. whether observed CP types are consistent with GCM-generated CPs, (3) sensitivity of results to the use of different GCMs. These problems have been addressed by, among others, Vaccaro (1992), Wilson et al. (1992), Matyasovszky et al. (1993b), and Matyasovszky \& Bogardi (1996).

\section{DISCUSSION AND CONCLUSIONS}

The research reported in this paper addressed 2 main questions: estimation of the probability distribution of daily global radiation, and development of a stochastic climatological model to describe the space-time behavior of daily global radiation.

In contrast to several climatic elements, no appropriate model is available for the probability distribution of radiation characteristics. In this paper a nonparametric technique, the Abramson kernel density estimator, was presented to estimate the probability density of global radiation. The densities are smooth enough to satisfy a subjective smoothness property required for any estimate of probability distributions, while they fit the histograms accurately. Kernel density estimators have both advantages and disadvantages. A disadvantage is that no simple estimation equation is available and the calculation of the density at any point requires the entire data set. An advantage is its high flexibility, not a single parametric distribution could produce such different densities than those shown in Fig. 4.

The space-time stochastic behavior of daily global radiation is described, after a transformation, by a multivariate autoregressive (AR) process. Conditioning on the types of large-scale circulation patterns results in quite different densities. An application of the model with a simulation procedure shows that both the spatial and temporal variability of daily solar radiation are reproduced.

The following conclusions can be drawn from this study:

(1) Due to the complex processes influencing solar radiation, common probability distributions can be rarely found to characterize daily radiation. To this end, flexible nonparametric approaches, e.g. the Abramson kernel method, can be used to estimate the probability distribution

(2) The space-time properties of daily global radiation can be described with a transformed autoregressive process. The transformation provides the relation- ship between daily global radiation and a normal random variable.

(3) Daily global radiation is shown to be highly dependent on the prevailing daily atmospheric circulation pattern. Thus, it makes sense to use the stochastic model of global radiation conditioned on CP types.

(4) Using a simulation procedure the stochastic climatological model reproduces the observed spacetime stochastic properties of daily global radiation at 7 locations in Nebraska.

(5) The space-time stochastic model can be used for downscaling of the low resolution output of GCMs.

Acknowledgements. Research leading to this paper has been partly supported by grants from the U.S. National Science Foundation, EAR-9205717/9217818, and the Great Plains Regional Center of the National Institute for Global Environmental Change. The additional support of the Center for Infrastructure Research of the University of Nebraska is also acknowledged

\section{LITERATURE CITED}

Bardossy A, Caspary H (1990) Detection of climate change in Europe by analyzing European circulation patterns from 1881 to 1989. Theor Appl Climatol 42:155-167

Bardossy A, Plate E (1990) Modeling daily rainfall using a semi-Markov representation of circulation pattern occurrence. J Hydrol 66:33-47

Bardossy A, Plate E (1992) Space-time model for daily rainfall using atmospheric circulation patterns. Water Resour Res 28:1247-1260

Baur F, Hess P, Nagel H (1944) Kalendar der Groswetterlagen Europas 1881-1939. Bad Homburg

Bogardi I, Matyasovszky I, Bardossy A, Duckstein L (1993) Application of a space-time stochastic model for daily precipitation using atmospheric circulation patterns. J Geophys Res 98(D9): 16653-16667

Craddock JM, Flood CR (1969) Eigenvectors for representing the $500 \mathrm{mb}$ geopotential surface over the Northern Hemisphere. Q J R. Meteor ol Soc 95:576-593

Dzerdzeevskii BL (1968) Circulation mechanusms in the atmosphere of the northern hemisphere in the twentieth century. Institute for Geography, Soviet Academy of Sciences, Moscow

Easterling DR (1995) Statistical generation of surface air temperature for the central USA using a general circulation. model simulation. Proceedings of 6th International Meeting on Statistical Climatology. University College, Galway, p 201-202

Essenwanger $O$ (1976) Applied statistics in atmospheric science. Part A. Frequencies and curve fitting. Elsevier, Amsterdam

Gasser T, Muller HG, Mammitzch V (1985) Kernels for nonparametric curve estimation. Q J R Statist Soc B 47: $238-252$

Giorgi, Mearns (1991) Approaches to the simulation of regional climate change: a review. Rev Geophys 29: $191-216$

Gong X, Richman MB (1992) An examination on methodological issues in clustering North American precipitation. Proceedings of 5 th International Meeting on Stochastic Climatology. Atmospheric Environment Service, Toronto, p J103-J108 
Hall P (1992) On global properties of variable bandwidth density estimators. Ann Stat 20:762-778

Hall P, Marron JS (1987) Extent to which least squares crossvalidation minimizes integrated squared error in nonparametric density estimation. Prob Theory Rel Fields 74 $567-568$

Hartigan J (1975) Clustering algorithms. Wiley. New York

Hess P. Brezowsky H (1969) Katalog der Grosswetterlagen Europas. Berichte des Deutchen Wetterdienstes Nr $113 \mathrm{Bd}$ 15 2, Neu bearbeitete und ergänzte Aufl., Offenbach a. Main

Key J, Crane RG (1986) A comparison of synoptic classification schemes based on 'objective procedures' J Climatol 6:375-386

Kirchofer W (1973) Classification of European $500 \mathrm{mb}$ patterns. Arbeitsbericht der Schweizerischen Meteorologischen Zentralanstalt No. 43, Geneva

Kutzbach JE (1970) Large-scale features of monthly mean Northern Hemisphere maps of sea-level pressure. Mon Weather Rev 98:708-721

Lamb HH (1972) British Isles weather types and a register of the daily sequence of circulation patterns, 1861-1971. (ieophys Mem. No 116, Indon

Lamb HH (1977) Climate, present, past and future Vol. 2: Climatic history and the future. Methuen \& Co Ltd, London

Leathers DJ (1991) Relationship between 700 mb circulation variation and Great Plains climate. Great Plain Res 1. $58-76$

Leathers DJ, Yarnal B, Palecki MA (1991) The Pacific/North American teleconnection pattern and United States climate. Part I: Regional temperature and precipitation associations. J Clim 4:517-528

Lund IA (1963) Map-pattern classification by statistical methods. J Appl Meteorol 2:56-65

MacQueen J (1967) Some methods for classification and analysis of multivariate observations. Proc 5th Berkeley Symp on Math Stat and Prob, Vol 1. University Press, Berkeley, p 281-297

Editor: V. Meentemeyer, Athens, Georgia, USA
Matyasovszky I, Bogardi I (1996) Downscaling two versions of a GCM to estimate local hydroclimatic factors under cl.tmate change. Hydrol Sci J 41:117-129

Matyasovszky I, Bogardi I, Bardossy A, Duckstein L (1993a) Space-time precipitation reflecting climate change. Hydrol Sci J 38:539-558

Matyasovszky I, Bogardi I. Bardossy A, Duckstein L (1993b) Estimation of local precipitation statistics reflecting climate change. Water Resour Res 29:3955-3968

Matyasovszky I, Bogardi I, Bardossy A, Duckstein L (1994) Local temperature estimation under climate change. Theor Appl Climatol 50:1-13

Muller HG (1984) Smooth optimum kernel estimates of densities, regression curves and modes. Ann Stat 12:766-774

Muller HG (1991) Smooth optimum kernel estimators near endpoints. Biometrika 78:521-530

Parzen $E$ (1962) On the estimation of probability density function and mode. Ann Math Stat 33:1065-1076

Priestly MB (1981) Spectral analysis and time series. Academic Press, New York

Rosenblatt M (1956) On some nonparametric estimates of a density function. Ann Math Stat 27:832-837

Rudemo M (1982) Empirical choice of histograms and kornel density estimators. Scand J Stat 9:65-78

Simmons AJ, Bengtsson L (1988) Atmospheric general circulation models: their design and use for climate studies. In: Schlesinger $M$ (ed) Physically-based modelling and simulation of climate and climatic change. NATO ASI Ser Kluwer Academic, Boston, p 627-652

Sowden IP, Parker DE (1981) A study of climatic variability of daily Central England temperatures in relation to the Lamb synoptic types. J Climatol 1:3-10

Vaccaro JJ (1992) Sensitivity of groundwater recharges estimates to climate variability and change, Columbia Plateau, Washington. J Geophys Res 97(D3):2821-2833

Wilson LL, Lettenmaier DP, Skyllingstad E (1992) A hierarchical stochastic model of large scale atmospheric circulation patterns and multiple station daily precipitation. I Geophys Res 97(D3):2791-2809

Manuscript first received: September 18, 1995

Revised version accepted: May 15, 1996 